\title{
Vancomycin-associated acute kidney injury in Hong Kong in 2012-2016
}

\author{
Xuzhen Qin ${ }^{1}$, Man-Fung Tsoi ${ }^{2}$, Xinyu Zhao ${ }^{3}$, Lin Zhang ${ }^{1}$, Zhihong Qi ${ }^{1}$ and Bernard M. Y. Cheung ${ }^{2,4^{*}}$ (D)
}

\begin{abstract}
Background: To study the incidence of vancomycin-associated acute kidney injury (VA-AKI) in Hong Kong and identify risk factors for VA-AKI.

Method: Patients with vancomycin prescription and blood level measurement in 2012-2016 were identified using the Hong Kong Hospital Authority Clinical Data Analysis and Reporting System. Acute kidney injury was defined using KDIGO criteria. Patients without creatinine measurements, steady-state trough vancomycin level or who had vancomycin treatment $<3$ days were excluded. Results were analyzed using SPSS version 22.0. Logistic regression was used to identify the predictors for VA-AKI. Odds ratio and 95\% confidence interval were estimated.

Results: One thousand four hundred fifty patients were identified as VA-AKI from 12,758 records in Hong Kong in 2012-2016. The incidence was respectively 10.6, 10.9, 11.3, 12.2, 11.2\% from 2012 to 2016. The incidence of VA-AKI was $16.3,12.2,11.3$ and $6.2 \%$ in patients aged 1-12, 12-60, elderly aged $>60$ and newborn and infants, respectively. Baseline creatinine, serum trough vancomycin level, systematic disease history including respiratory failure, hypertension, congestive heart failure, chronic renal failure, anemia and type II diabetes, and concomitant diuretics, piperacillin-tazobactam (PTZ) and meropenem prescription were significantly higher in VA-AKI patients older than 12 years. Logistic regression showed that older age group, higher baseline creatinine, serum trough vancomycin level, respiratory failure, chronic renal failure and congestive heart failure, concomitant diuretics, PTZ and meropenem prescription, and longer hospital stay were all associated with increased risk of VA-AKI.
\end{abstract}

Conclusion: The incidence of VA-AKI in Hong Kong is low but shows no decline. Patients with higher baseline creatinine, multi-organ diseases and multiple drugs administration should have their vancomycin level monitored to decrease the risk of VA-AKI.

Keywords: Vancomycin, Acute kidney injury

\section{Background}

Methicillin-resistant Staphylococcus aureus (MRSA) has gained considerable attention due to its high morbidity, limited effective treatments available and increased incidence [1]. Vancomycin has been recommended as the first line treatment for MRSA infections by American, European, and Chinese guidelines for infectious diseases control and management control for antibiotics [2-5]. However, the significant nephrotoxicity limits the use of vancomycin in daily clinical practice. It has been

\footnotetext{
* Correspondence: mycheung@hku.hk

Department of Medicine, The University of Hong Kong, Hong Kong, China

${ }^{4}$ State Key Laboratory of Pharmaceutical Biotechnology, The University of Hong Kong, Hong Kong, China

Full list of author information is available at the end of the article
}

reported the incidence of VA-AKI ranges from $0 \%$ to over $40 \%$ [6].

AKI is one of the examples of nephrotoxicity that accounts for about 1.7 million deaths every year worldwide. The consequences of AKI can be severe, ranging from hospitalization to life-long dialysis and transplantation. From a recent meta-analysis, the pooled incidence of AKI was 19.4\% in eastern Asia [7]. Drugs are the third main cause of AKI [8]. The prognosis in drug-associated AKI is poor, especially in critically ill patients.

In Hong Kong, there is limited published literature on the incidence of drug-induced nephrotoxicity. A previous study reported an incidence of $23.1 \%$ for VA-AKI in a hospital [9]. A systematic population-wide study is clearly needed, in view of the serious implications of this

(c) The Author(s). 2020 Open Access This article is distributed under the terms of the Creative Commons Attribution 4.0 International License (http://creativecommons.org/licenses/by/4.0/), which permits unrestricted use, distribution, and 
potentially preventable and reversible condition. Therefore, we investigated the incidence of VA-AKI in Hong Kong from 2012 to 2016, characterized patients with VA-AKI as well as identified predictors for the development of VA-AKI.

\section{Methods}

\section{Study design and data sources}

A retrospective study was conducted using Clinical Data Analysis and Report System (CDARS), a database managed by the Hong Kong Hospital Authority (HA). HA is the sole public hospital service provider in Hong Kong. More than $90 \%$ of Hong Kong residents use the public hospital services [10]. CDARS captures patient records in Hong Kong in all public hospitals and outpatient clinics since 1993. This database provides a convenient platform for pharmaco-epidemiological studies [11].

All patients' records with vancomycin prescription and measurement of serum drug level from January 2012 to December 2016 in Hong Kong were retrieved from CDARS. Demographics, laboratory parameters, concomitant medications and co-morbidities were collected as the covariates. Patients with vancomycin prescription and steady-state serum trough vancomycin level were included as the exposure status. Patients who had no baseline and follow-up concentration of creatinine within 48 $h$, vancomycin treatment $<3$ days or steady state serum trough vancomycin level were excluded. Trough concentrations were defined as the results from the samples taken within $1 \mathrm{~h}$ of the next due dose. The first trough concentration was measured after 3 days of vancomycin administration.

\section{Definition of AKI}

With the use of Kidney Disease Improving Global Outcomes (KDIGO) criteria [12], patients with AKI were identified as increase in serum creatinine $(\mathrm{SCr})$ by 0.3 $\mathrm{mg} / \mathrm{dL}$ or more within $48 \mathrm{~h}$, or as a $1.5 \sim 1.9$ times increase in $\mathrm{SCr}$ from baseline. These patients were at least of stage I in severity. Stage II was defined as 2 2.9 times increase in $\mathrm{SCr}$ from baseline. Stage III was defined as 3 times increase in $\mathrm{SCr}$ from baseline or increase in $\mathrm{SCr}$ to $\geq 4 \mathrm{mg} / \mathrm{dL}$. Vancomycin-associated AKI was the main outcome for the study. Patients without VA-AKI were classified as non-VA-AKI group.

\section{Statistical analysis}

IBM SPSS statistics version 22.0 (IBM SPSS Statistics, Armonk, NY, USA) was used for data analysis. Descriptive statistics were expressed as frequency for categorical data or median (interquartile range) for continuous variables. With reference to a vancomycin therapeutic guideline [5], vancomycin concentration was categorized into 4 groups: < $10.0 \mathrm{mg} / \mathrm{L}, 10.0 \sim 15.0 \mathrm{mg} / \mathrm{L}, 15.0 \sim 20.0$ $\mathrm{mg} / \mathrm{L},>20.0 \mathrm{mg} / \mathrm{L}$. Continuous variables were compared among groups using one-way ANOVA and KruskalWallis tests as appropriate. Categorical variables were compared among groups using Pearson Chi-square test. Logistic regression was used to estimate odd ratios (ORs) and 95\% confidence interval (CI). Firstly, univariate logistic models were used to explore the relationship between VA-AKI and risk factors, including gender, age, route of administration, baseline creatinine, tough vancomycin level, concomitant drugs, length of stay and death in hospital. Secondly, to determine whether there was significant collinearity between continuous variables, collinearity diagnostics were performed using variance inflation factor (VIF) and tolerance values. If tolerance value is close to zero or VIF is greater than 10, there may be multi-collinearity. Thirdly, four models adjusted by different confounders were applied to analyse the possible predicators. Model 1: adjusted for demographics (age, sex); Model 2: additionally adjusted for comorbidities; Model 3: additionally adjusted for medications; Model 4: additionally adjusted for baseline creatinine and length of stay. Predictor variables were entered if $P<0.05$ and removed if $P>0.10$ in the stepwise model. A two-tailed $\mathrm{P}<0.05$ was considered statistically significant. A two-tailed $\mathrm{P}<0.05$ will be considered statistically significant.

\section{Results}

A total of 12,758 patients were included in this study from 2012 to 2016 in Hong Kong (Fig. 1). The median age of patients with VA-AKI was 72 years old, and $64.2 \%$ of cases were male. The prescription of vancomycin increased gradually and doubled in 2016 compared with 2012. The incidence of VA-AKI remained at approximately $11.4 \%$ (Table 1 ).

Table 2 shows the characteristics of patients with VAAKI stratified by age group. The incidence of VA-AKI in patients aged 1 to 12 was the highest (16.3\%), following patients aged 12 to 60 (12.2\%), elderly aged over 60 (11.3\%) and newborn and infants (6.2\%). The median age of VA-AKI patients was slightly younger than patients without VA-AKI in elderly group, yet older than patients without VA-AKI in patients less than 60 years old. There was no significant gender difference between VA-AKI and non-VA-AKI groups in all age groups. The main route of administration was injection and parenteral, with several cases of oral, eye drop and intraperitoneal route. The incidence of VA-AKI was distinctively higher in newborn and infants receiving parenteral administration $(P<0.001)$. The baseline creatinine and trough vancomycin concentration in the VA-AKI group were significantly higher than non-VA-AKI group in patients older than 12 years old $(P<0.001)$. However, there were no significant difference in patients aged under 12 . 


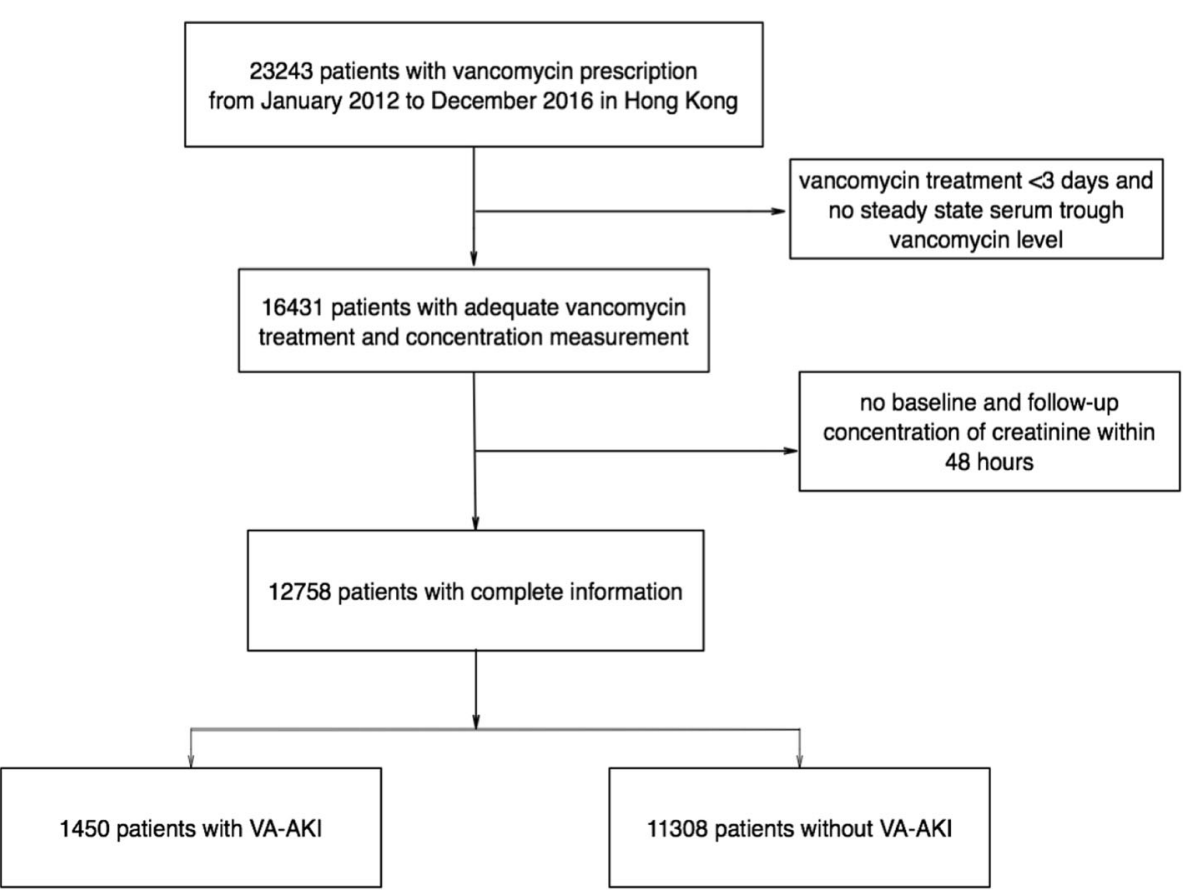

Fig. 1 A flow diagram for inclusion of study samples

Along with the increase of trough vancomycin level, the incidence of VA-AKI either in cumulative incidence or in each AKI stage showed a significantly upward trend $(P<0.001)$ (Fig. 2). Compared to non-VA-AKI group, more patients with VA-AKI aged over 12 had comorbidities including respiratory failure, essential hypertension, congestive heart failure, chronic renal failure, anaemia and type II DM. There were more comorbidities in VAAKI patients between 12 to 60 years old compared with non-VA-AKI group in the same age group. Patients with VA-AKI aged over 12 were more likely to receive diuretics, piperacillin-tazobactam (PTZ) and meropenem, while more angiotensin converting enzyme inhibitors (ACEI) prescripts were found in patients with VA-AKI from 1 to 12 years old, more ACEI, angiotensin II receptor blocker (ARB), non-steroidal anti-inflammatory drugs (NSAID) prescripts in VA-AKI group from 12 to
60 years old, and more aminoglycoside and amphotericin $B$ prescripts in VA-AKI patients over 60 years old. Patients over 60 years old with VA-AKI were hospitalized for longer duration. VA-AKI group had a significantly higher mortality in hospital compared with non-VA-AKI group.

Logistic analysis shows the possible risk factors associated with VA-AKI (Table 3). Using both univariate logistic model and adjusted logistic models showed that older age, baseline creatinine, trough vancomycin level, the comorbidities including essential hypertension, anaemia, respiratory failure, type II DM, chronic renal failure and congestive heart failure, concomitant drugs including diuretics, ACEI, ARB, PTZ, and meropenem were all associated with higher risk for the development of VA-AKI. Regarding collinearity, the highest VIF and the lowest tolerance values were 1.016 and 0.87 ,

Table 1 Incidence and demographic characters of patients with VA-AKI in Hong Kong from 2012 to 2016

\begin{tabular}{|c|c|c|c|c|c|}
\hline & 2012 & 2013 & 2014 & 2015 & 2016 \\
\hline Annual admissions & $1,466,788$ & $1,508,489$ & $1,532,926$ & $1,592,148$ & $1,629,346$ \\
\hline Vancomycin prescription & 3310 & 3510 & 4132 & 5597 & 6694 \\
\hline Enrolled patients & 1542 & 1709 & 2446 & 3374 & 3687 \\
\hline Male & $63.1 \%$ & $62.5 \%$ & $62.9 \%$ & $63.7 \%$ & $64.0 \%$ \\
\hline Female & $36.9 \%$ & $37.5 \%$ & $37.1 \%$ & $36.3 \%$ & $36.0 \%$ \\
\hline Age & $71.7(52.8,83.0)$ & $71.6(55.8,81.9)$ & $73.8(58.5,83.4)$ & $71.8(58.1,83.5)$ & $76.5(62.1,85.7)$ \\
\hline VA-AKI cases & 164 & 186 & 276 & 410 & 414 \\
\hline Incidence of VA-AKI & $10.6 \%$ & $10.9 \%$ & $11.3 \%$ & $12.2 \%$ & $11.2 \%$ \\
\hline
\end{tabular}




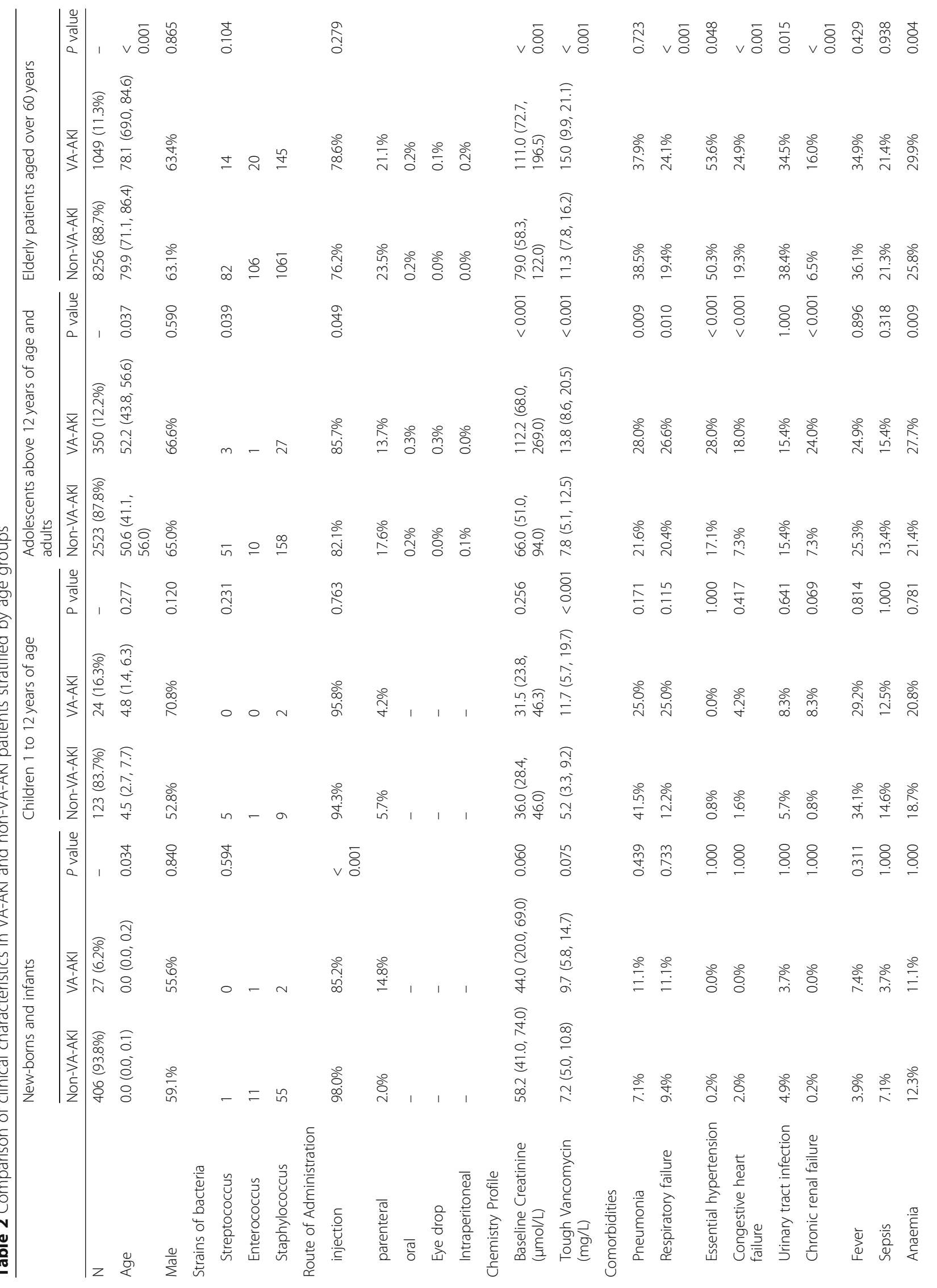




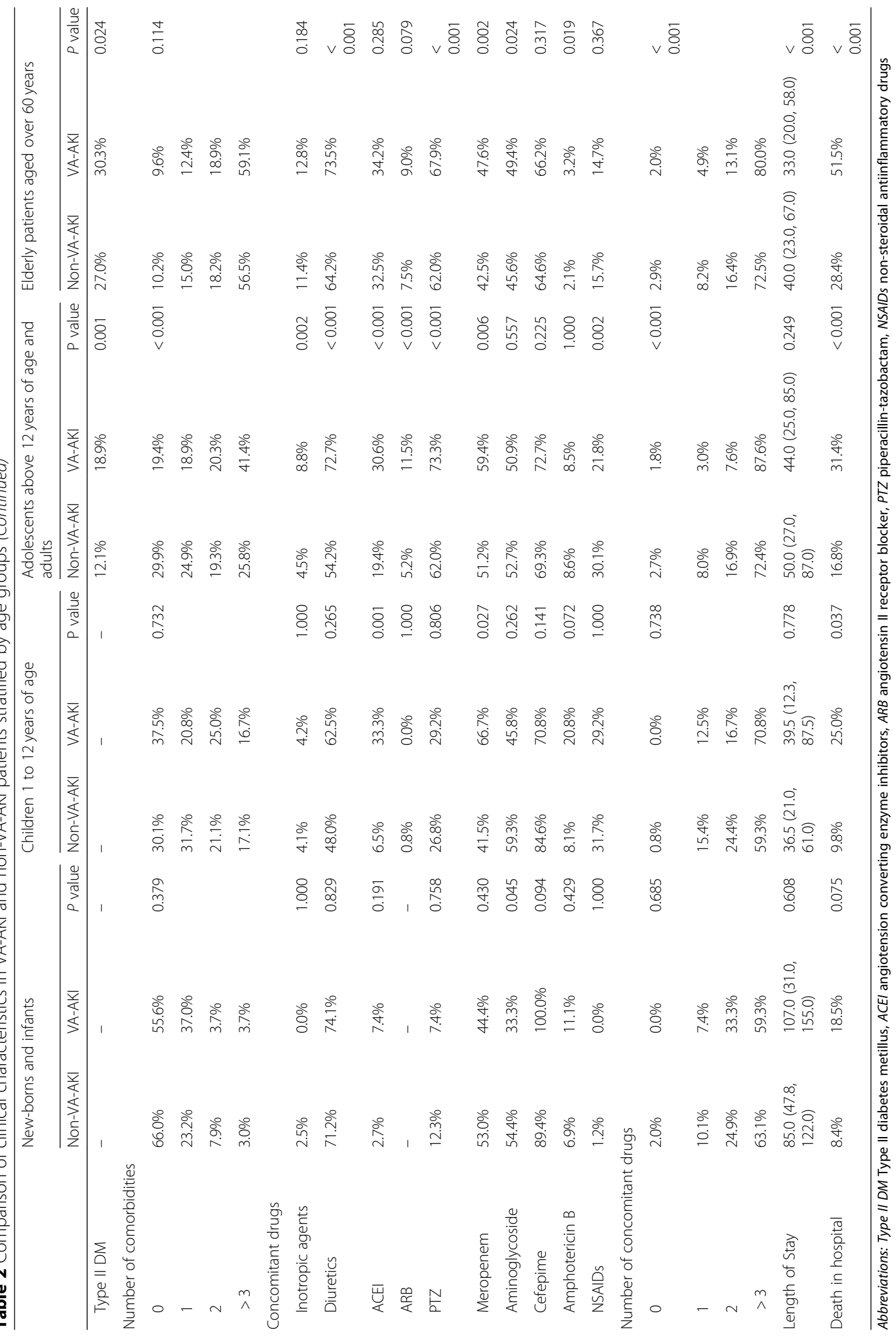




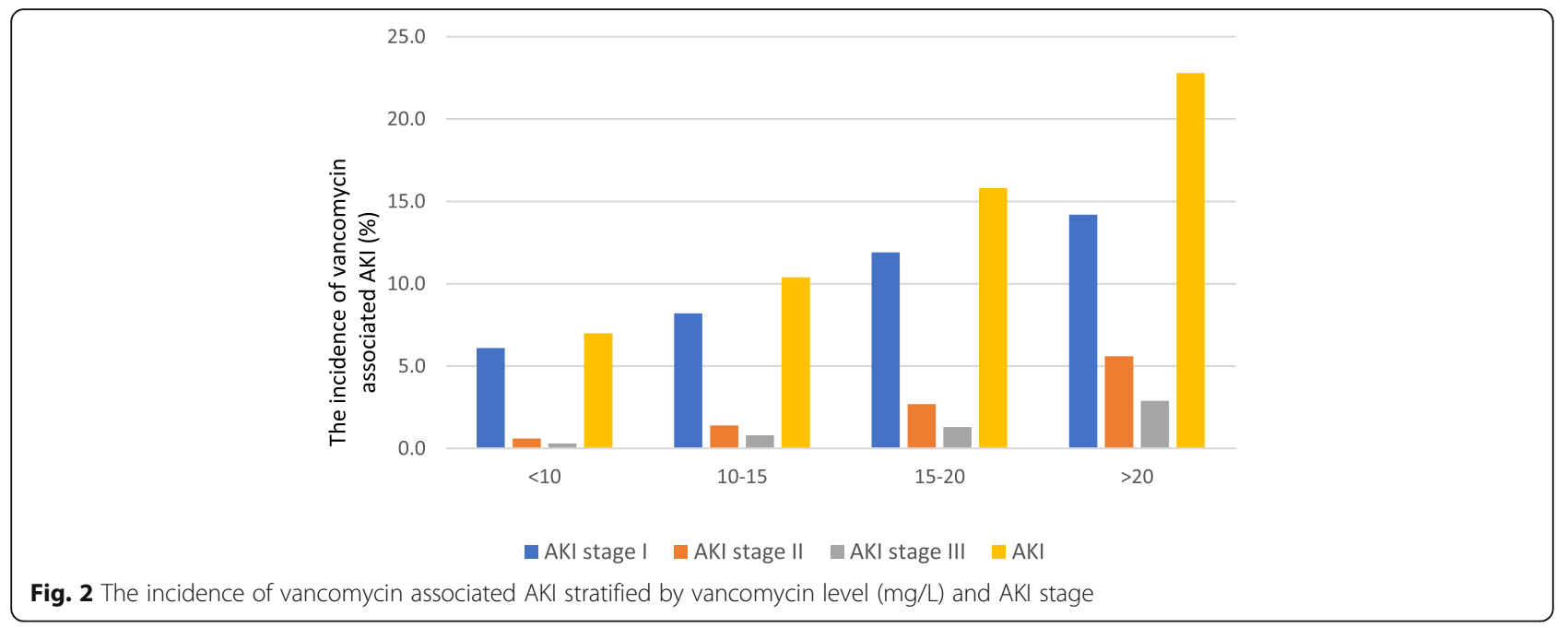

respectively, which suggests the absence of significant collinearity. Compared with newborns and infants, older age groups had lower ORs for development of VA-AKI (OR of children 1 to 12 years of age: 2.93 (95\%CI [1.63, 5.27], OR of adults and adolescents above 12 years of age: 2.09 (95\%CI [1.39, 3.13], OR of elderly patients: 1.91 (95\%CI [1.29, 2.84], respectively). Most of these predicators had positive relationships with VA-AKI, yet, urinary tract infection had a negative relationship with VA-AKI (OR: 0.75 (95\%CI [0.66, 0.86]). After adjusted for gender, age, comorbidities, concomitant drugs, baseline creatinine and length of stay in various models with increasing confounders, vancomycin, especially vancomycin concentration over 10 were still significant predicators for VA-AKI. Compared to the vancomycin level less than $10.0 \mathrm{mg} / \mathrm{L}$ group, higher vancomycin level had higher OR for VA-AKI onset in univariate logistic model (OR of 10.0 15.0 level: 1.55 (95\%CI [1.34, 1.81], OR of 15.1 20.0 level: 2.50 (95\%CI [2.13, 2.93], OR of $>20.0$ level: 3.89 (95\%CI [3.34, 4.53], respectively).

\section{Discussion}

In this study involving 12,578 patients on vancomycin, the incidence of VA-AKI was $11.4 \%$ in Hong Kong in 2012-2016. Compared with non-VA-AKI group, VAAKI patients older than 12 years had significant higher level or frequency in the baseline creatinine, trough vancomycin level, systematic disease history including respiratory failure, essential hypertension, congestive heart failure, chronic renal failure, anaemia and type II $\mathrm{DM}$, and concomitant drugs usage including diuretics, PTZ and meropenem. VA-AKI group had a significantly higher mortality in hospital compared with non-VA-AKI group. The incidence of VA-AKI increased with increased vancomycin level. Stepwise logistic regression showed that older age group, higher vancomycin level, comorbidities including respiratory failure, chronic renal failure and congestive heart failure, concomitant drugs including diuretics, PTZ and meropenem and length of stay were all independent risk factors for the development of VA-AKI.

This study is the first large scale investigation of VAAKI in Hong Kong. In previous studies, the incidence of VA-AKI in Chinese varied from 2.4 to $45 \%$ [9, 13-16] (Additional file 1: Table S1). The difference of estimated incidence is due to different populations, sample sizes, inclusion criteria and AKI definition. In USA, the incidence of VA-AKI declined from $42.6 \%$ in 2007 to $14.1 \%$ in 2017 [17, 18]. This might be due to doctors' awareness of the toxicity of vancomycin and improvements in vancomycin purity. Our study reports a lower incidence of VA-AKI than the previous study [9], since the large database is less prone to publication and other biases.

In the literature, nephrotoxicity occurs in $1-9 \%$ of neonates receiving currently recommended doses, and $6-14 \%$ of paediatric patients [19-21]. Since these studies were all based on different population, the incidence of VA-AKI cannot be compared. Our study compared the incidence of VA-AKI in different age group based on the same population. By age group, the order of incidence from high to low was children from 1 to 12 years old (16.3\%), adolescents above 12 years of age and adults (12.2\%), elderly patients aged over 60 years (11.3\%), newborns and infants (6.2\%). The characters of young patients with VA-AKI were different from elderly patients. The median of trough vancomycin in patients under 60 years old were all lower than $10 \mathrm{mg} / \mathrm{L}$, yet the incidence of VA-AKI still accounted for a sizeable proportion. In new-borns and infants receiving parenteral administration the incidence of VA-AKI was distinctively higher.

There have been studies on the risk factors for VAAKI in the past [22-24]. Like previous studies, a positive correlation between VA-AKI and increasing vancomycin trough concentrations and baseline serum creatinine was 
Table 3 Predicators for VA-AKI in Hong Kong

\begin{tabular}{|c|c|c|c|c|c|}
\hline & OR & Model 1 & Model 2 & Model 3 & Model 4 \\
\hline Male & $0.96(0.86,1.08)$ & - & - & - & - \\
\hline \multicolumn{6}{|l|}{ Age } \\
\hline Newborn and infants & Reference & - & - & - & - \\
\hline Children 1 to 12 years of age & $2.93(1.63,5.27)^{*}$ & - & - & - & - \\
\hline Adults and adolescents above 12 years of age & $2.09(1.39,3.13)^{*}$ & - & - & - & - \\
\hline Elderly patients & $1.91(1.29,2.84)$ & - & - & - & - \\
\hline \multicolumn{6}{|l|}{ Chemistry profile } \\
\hline Baseline creatinine & $1.00(1.00,1.00)^{*}$ & $1.00(1.00,1.00)^{*}$ & $1.00(1.00,1.00)^{*}$ & $1.00(1.00,1.00)^{*}$ & - \\
\hline Trough vancomycin level & $1.04(1.04,1.05)^{*}$ & $1.05(1.04,1.05)^{*}$ & $1.04(1.04,1.05)^{*}$ & $1.04(1.04,1.05)^{*}$ & $1.04(1.04,1.05)^{*}$ \\
\hline \multicolumn{6}{|l|}{ Classification of vancomycin level (mg/L) } \\
\hline$<10.0$ & Reference & Reference & Reference & Reference & Reference \\
\hline $10.0 \sim 15.0$ & $1.55(1.34,1.81)^{*}$ & $1.68(1.44,1.96)^{*}$ & $1.63(1.39,1.92)^{*}$ & $1.62(1.38,1.89)^{*}$ & $1.64(1.40,1.93)^{*}$ \\
\hline $15.1 \sim 20.0$ & $2.50(2.13,2.93)^{*}$ & $2.75(2.33,3.24)^{*}$ & $2.62(2.21,3.11)^{*}$ & $2.61(2.20,3.08)^{*}$ & $2.62(2.21,3.11)^{*}$ \\
\hline$>20.0$ & $3.89(3.34,4.53)^{*}$ & $4.29(3.67,5.02)^{*}$ & $3.93(3.34,4.63)^{*}$ & $4.05(3.45,4.77)^{*}$ & $4.07(3.46,4.79)^{*}$ \\
\hline \multicolumn{6}{|l|}{ Comorbidities } \\
\hline Pneumonia & $1.06(0.94,1.19)$ & $1.07(0.96,1.21)$ & - & - & - \\
\hline Essential hypertension & $1.23(1.10,1.38)^{*}$ & $1.33(1.18,1.50)^{*}$ & - & & - \\
\hline Urinary tract infection & $0.87(0.77,0.98) \#$ & $0.88(0.77,1.00)$ & - & - & - \\
\hline Fever & $0.97(0.86,1.09)$ & $0.98(0.87,1.11)$ & - & - & - \\
\hline Anemia & $1.29(1.14,1.45)^{*}$ & $1.30(1.15,1.48)^{*}$ & - & - & - \\
\hline Respiratory failure & $1.39(1.22,1.58)^{*}$ & $1.39(1.22,1.58)^{*}$ & - & - & - \\
\hline Type II DM & $1.25(1.10,1.41)^{*}$ & $1.28(1.13,1.46)^{*}$ & - & - & - \\
\hline Sepsis & $1.03(0.90,1.19)$ & $1.05(0.91,1.20)$ & - & & - \\
\hline Chronic renal failure & $3.17(2.72,3.70)^{*}$ & $3.18(2.72,3.71)^{*}$ & - & - & - \\
\hline Congestive heart failure & $1.56(1.37,1.79)^{*}$ & $1.63(1.42,1.87)^{*}$ & - & - & - \\
\hline \multicolumn{6}{|l|}{ Concomitant drugs } \\
\hline Inotropic agents & $1.26(1.05,1.50) \#$ & $1.28(1.07,1.53) \#$ & $1.19(0.99,1.43)$ & - & - \\
\hline Diuretics & $1.71(1.51,1.94)^{*}$ & $1.72(1.51,1.95)^{*}$ & $1.49(1.31,1.70)^{*}$ & & - \\
\hline ACEl & $1.25(1.11,1.41)^{*}$ & $1.27(1.12,1.43)^{*}$ & $1.11(0.98,1.27)$ & - & - \\
\hline ARB & $1.46(1.20,1.77)^{*}$ & $1.47(1.21,1.79)^{*}$ & $1.15(0.94,1.42)$ & - & - \\
\hline PTZ & $1.39(1.24,1.57)^{*}$ & $1.41(1.25,1.59)^{*}$ & $1.39(1.23,1.57)^{*}$ & - & - \\
\hline Meropenem & $1.29(1.16,1.45)^{*}$ & $1.29(1.15,1.44)^{*}$ & $1.23(1.10,1 \cdot 39)^{*}$ & - & - \\
\hline Aminoglycoside & $1.71(0.96,1.22)$ & $1.07(0.95,1.21) \#$ & $1.05(0.94,1.18)$ & - & - \\
\hline Cefepime & $1.31(1.01,1.71)$ & $1.29(0.99,1.69)$ & $1.04(0.92,1.18)$ & & - \\
\hline Amphotericin B & $0.84(0.73,0.98) \#$ & $0.84(0.72,0.97)$ & $1.24(0.95,1.63)$ & - & - \\
\hline NSAIDs & $1.00(1.00,1.00) \#$ & $1.00(1.00,1.00)$ & $0.93(0.80,1.09)$ & - & - \\
\hline Length of Stay & $0.97(0.86,1.09)$ & $0.98(0.87,1.11)$ & $1.00(1.00,1.00)$ & $1.00(1.00,1.00) \#$ & - \\
\hline
\end{tabular}

Data is expressed as odds ratio (95\% confidence interval)

-Not available or not in the equation

Model 1: adjusted for age and gender

Model 2: additionally, adjusted for comorbidities;

Model 3: additionally, adjusted for medications;

Model 4: additionally, adjusted for baseline creatinine and length of stay

${ }^{*} p<0.001 \# p<0.05$ 
found in this study. The association between age and VA-AKI was controversial, mostly with a positive correlation [24]. While some studies explored after controlling for comorbid condition, medications and renal function, age is not associated with AKI [25]. In this study, we found age was correlated with VA-AKI, yet compared with new-borns and infants age group, older age group had less OR for development of VA-AKI. Concomitant administration of diuretics, PTZ and meropenem could increase the incidence of nephrotoxicity [22, 26, 27], except concomitant NSAIDs seems to have a protective role for VA-AKI. In each age group, VA-AKI incidence was lower in patients with concomitant NSAIDs. We confirmed that concomitant chronic renal failure, congestive heart failure and respiratory failure increased the risk for VA-AKI, as in a previous study in China [15]. Although vancomycin administrated by eye-drops, oral and intraperitoneal has low bioavailability, a previous study reported AKI development was related with the usage of topical fortified gentamicin and vancomycin eyedrops [28]. Similarly, serum concentration of vancomycin could also be observed in VA-AKI group in different routes of administration in this study. The incidence and mechanism of VA-AKI in uncommon routes of administration remains uncertain, yet the possible risk for AKI development should be noticed.

Several explanations have been put forth on the cause of VA-AKI, including impurities in vancomycin [29], critically-ill patients with complications and on multiple drugs, and lack of suitable control patients. A randomized controlled trial was conducted to identify the relationship between vancomycin and the development of AKI. Patients were randomized to continuation of doseoptimized vancomycin or early switch to an alternative antimicrobial agent. The incidence of AKI in both groups was similar (32.7 to $31.4 \%[P=0.89]$ ) [30], but this was a small study of 103 patients in a single center.

The strength of this study was that it was populationbased and territory-wide, and used a very large database. In the limited literature, the incidence of AKI in Hong Kong was reported as $11.7 \%$ [31], similar to the incidence of VA-AKI in our study. It provides good evidence that dosing and monitoring practices of vancomycin in Hong Kong are sound. However, there were limitations. Since it was an observational study, causation cannot be proven. Patients with VA-AKI could have other reasons for AKI, such as comorbidities, hypotension, sepsis and concomitant drugs. It is distinctly difficult to determine the actual risk factors of VA-AKI.

\section{Conclusion}

In summary, the incidence of VA-AKI in Hong Kong is low but shows no decline. As there is increased usage of vancomycin to combat MRSA, there should be increased awareness of VA-AKI. Patients with higher baseline creatinine, chronic diseases and multiple drugs are at risk of VA-AKI and should have their vancomycin level monitored. Our findings facilitate the development of strategies and guidelines to prevent vancomycinassociated AKI.

\section{Supplementary information}

Supplementary information accompanies this paper at https://doi.org/10. 1186/s12882-020-1704-4

Additional file 1: Table S1. Literature review of incidence of VA-AKI in Chinese. The key points of previous publications regarding to VA-AKI in Chinese were summarized including the region, patients, definition of $\mathrm{AKI}$, incidence of VA-AKI and references.

\section{Abbreviations}

ACEl: Angiotension converting enzyme inhibitors; ARB: Angiotensin II receptor blocker; CDARS: Clinical Data Analysis and Report System;

Cl: Confidence interval; HA: Hong Kong Hospital Authority; KDIGO: Kidney Disease Improving Global Outcomes; MRSA: Methicillin-resistant

Staphylococcus aureus; NSAID: Non-steroidal anti-inflammatory drugs; ORs: Odd ratios; PTZ: Qpiperacillin-tazobactam; SCr: Serum creatinine; VAAKI: Vancomycin-associated acute kidney injury

\section{Acknowledgements}

QX was a recipient of a Dr. Cheng Yu Tung Fellowship.

\section{Authors' contributions}

QX made substantial contributions to conception, design and drafting the manuscript. $X Y Z, L Z$ and $Z H Q$ were involved in revising and drafting the manuscript for important intellectual content. MFT and BMYC made substantial contributions to conception and design, acquisition of data and revising of the final version of the manuscript. All authors have approved the final version of the manuscript and its conclusions, and take public responsibility for the accuracy and integrity.

\section{Funding}

No available funding.

\section{Availability of data and materials}

The datasets used and/or analysed during the current study available from CDARS on reasonable request.

\section{Ethics approval and consent to participate}

This project has been approved by Institutional Review Board of the University of Hong Kong/Hospital Authority Hong Kong West Cluster (HKU/ HA HKW IRB Reference Number: UW 19-378). This is a retrospective study and all patients included were identified anonymously.

\section{Consent for publication}

The authors all consent for publication.

\section{Competing interests}

The authors declare that they have no competing interests.

\section{Author details}

${ }^{1}$ Department of Laboratory Medicine, Chinese Academy of Medical Sciences \& Peking Union Medical College Hospital, Beijing, China. ${ }^{2}$ Department of Medicine, The University of Hong Kong, Hong Kong, China. ${ }^{3}$ Department of epidemiology and health statistics, Institute of basic medicine, Peking Union Medical College, Beijing, China. ${ }^{4}$ State Key Laboratory of Pharmaceutical Biotechnology, The University of Hong Kong, Hong Kong, China. 
Received: 19 September 2019 Accepted: 24 January 2020

Published online: 03 February 2020

\section{References}

1. Liu C, Bayer A, Cosgrove SE, et al. Clinical practice guidelines by the infectious diseases society of america for the treatment of methicillinresistant Staphylococcus aureus infections in adults and children: executive summary. Clin Infect Dis. 2011;52(3):285-92.

2. Ye ZK, Chen YL, Chen $\mathrm{K}$, et al. Therapeutic drug monitoring of vancomycin: a guideline of the division of therapeutic drug monitoring, Chinese Pharmacological Society. J Antimicrob Chemother. 2016;71(11):3020-5.

3. Chastre J, Blasi F, Masterton RG, Rello J, Torres A, Welte T. European perspective and update on the management of nosocomial pneumonia due to methicillin-resistant Staphylococcus aureus after more than 10 years of experience with linezolid. Clin Microbiol Infect. 2014;20(Suppl 4):19-36.

4. Mensa J, Soriano A, Llinares $P$, et al. Guidelines for antimicrobial treatment of the infection by Staphylococcus aureus. Rev Esp Quimioter. 2013;26(Suppl 1):1-84.

5. Rybak MJ, Lomaestro BM, Rotschafer JC, et al. Vancomycin therapeutic guidelines: a summary of consensus recommendations from the infectious diseases Society of America, the American Society of Health-System Pharmacists, and the society of infectious diseases pharmacists. Clin Infect Dis. 2009;49(3):325-7

6. Filippone EJ, Kraft WK, Farber JL. The nephrotoxicity of Vancomycin. Clin Pharmacol Ther. 2017;102(3):459-69.

7. Mehta RL, Cerdá J, Burdmann EA, et al. International society of Nephrology's Oby25 initiative for acute kidney injury (zero preventable deaths by 2025): a human rights case for nephrology. Lancet. 2015;385(9987):2616-43.

8. Uchino $S$. The epidemiology of acute renal failure in the world. Curr Opin Crit Care. 2006;12(6):538-43.

9. You JH, Chow SS, Lui JW, Ip M. New target concentrations for vancomycin in Hong Kong. Int J Antimicrob Agents. 2011;37(1):83-4

10. Mok CC, Kwok CL, Ho LY, Chan PT, Yip SF. Life expectancy, standardized mortality ratios, and causes of death in six rheumatic diseases in Hong Kong, China. Arthritis Rheum. 2011;63(5):1182-9.

11. Sing CW, Wong IC, Cheung BM, Chan JC, Chu JK, Cheung CL. Incidence and risk estimate of drug-induced agranulocytosis in Hong Kong Chinese. A population-based case-control study. Pharmacoepidemiol Drug Saf. 2017; 26(3):248-55.

12. Ad-hoc working group of $E$, Fliser $D$, Laville $M$, et al. A European renal best practice (ERBP) position statement on the kidney disease improving global outcomes (KDIGO) clinical practice guidelines on acute kidney injury: part 1: definitions, conservative management and contrast-induced nephropathy. Nephrol Dial Transplant. 2012;27(12):4263-72.

13. Dong MH, Wang JW, Wu Y, Chen BY, Yu M, Wen AD. Evaluation of body weight-based vancomycin therapy and the incidence of nephrotoxicity: a retrospective study in the northwest of China. Int J Infect Dis. 2015;37:125-8.

14. Wei WX, Qin XL, Cheng DH, Lu H, Liu TT. Retrospective analysis of vancomycin treatment outcomes in Chinese paediatric patients with suspected gram-positive infection. J Clin Pharm Ther. 2016;41(6):650-6.

15. Liu Y, Yin Y, Liu XZ, et al. Retrospective analysis of Vancomycin nephrotoxicity in elderly Chinese patients. Pharmacology. 2015;95(5-6):279-84.

16. Shen WC, Chiang YC, Chen HY, et al. Nephrotoxicity of vancomycin in patients with methicillin-resistant Staphylococcus aureus bacteraemia. Nephrology (Carlton). 2011;16(8):697-703.

17. Jeffres MN, Isakow W, Doherty JA, Micek ST, Kollef MH. A retrospective analysis of possible renal toxicity associated with vancomycin in patients with health care-associated methicillin-resistant Staphylococcus aureus pneumonia. Clin Ther. 2007;29(6):1107-15.

18. Rutter WC, Burgess DR, Talbert JC, Burgess DS. Acute kidney injury in patients treated with vancomycin and piperacillin-tazobactam: a retrospective cohort analysis. J Hosp Med. 2017;12(2):77-82

19. Lestner JM, Hill LF, Heath PT, Sharland M. Vancomycin toxicity in neonates: a review of the evidence. Curr Opin Infect Dis. 2016;29(3):237-47.

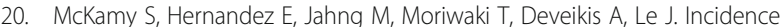
and risk factors influencing the development of vancomycin nephrotoxicity in children. J Pediatr. 2011:158(3):422-6.

21. Abouelkheir M, Alsubaie S. Pediatric acute kidney injury induced by concomitant vancomycin and piperacillin-tazobactam. Pediatr Int. 2018; 60(2):136-41.
22. Choi YC, Saw S, Soliman D, et al. Intravenous Vancomycin associated with the development of nephrotoxicity in patients with class III obesity. Ann Pharmacother. 2017;51(11):937-44.

23. Bhargava V, Malloy M, Fonseca $R$. The association between vancomycin trough concentrations and acute kidney injury in the neonatal intensive care unit. BMC Pediatr. 2017;17(1):50.

24. Conway EL, Sellick JA, Horey A, et al. Decreased mortality in patients prescribed vancomycin after implementation of antimicrobial stewardship program. Am J Infect Control. 2017;45(11):1194-7.

25. Carreno JJ, Jaworski A, Kenney RM, Davis SL. Comparative incidence of nephrotoxicity by age group among adult patients receiving Vancomycin. Infect Dis Ther. 2013;2(2):201-8.

26. Mellen CK, Ryba JE, Rindone JP. Does Piperacillin-Tazobactam increase the risk of nephrotoxicity when used with Vancomycin: a meta-analysis of observational trials. Curr Drug Saf. 2017:12(1):62-6.

27. Gomes DM, Smotherman C, Birch A, et al. Comparison of acute kidney injury during treatment with vancomycin in combination with piperacillintazobactam or cefepime.Pharmacotherapy. 2014;34(7):662-9.

28. Tang RK, Tse RK. Acute renal failure after topical fortified gentamicin and vancomycin eyedrops. J Ocul Pharmacol Ther. 2011:27(4):411-3.

29. Nambiar S, Madurawe RD, Zuk SM, et al. Product quality of parenteral vancomycin products in the United States. Antimicrob Agents Chemother. 2012;56(6):2819-23.

30. Carreno JJ, Kenney RM, Divine G, Vazquez JA, Davis SL. Randomized controlled trial to determine the efficacy of early switch from Vancomycin to Vancomycin alternatives as a strategy to prevent nephrotoxicity in patients with multiple risk factors for adverse renal outcomes (STOP-NT). Ann Pharmacother. 2017;51(3):185-93.

31. Szeto CC. Perspectives on acute kidney injury strategy: Hong Kong. Nephrology (Carlton). 2018;23(Suppl 4):104-6.

\section{Publisher's Note}

Springer Nature remains neutral with regard to jurisdictional claims in published maps and institutional affiliations.
Ready to submit your research? Choose BMC and benefit from:

- fast, convenient online submission

- thorough peer review by experienced researchers in your field

- rapid publication on acceptance

- support for research data, including large and complex data types

- gold Open Access which fosters wider collaboration and increased citations

- maximum visibility for your research: over $100 \mathrm{M}$ website views per year

At BMC, research is always in progress.

Learn more biomedcentral.com/submissions 